\title{
Satisfacción de los egresados con la formación recibida en el Máster de Estudios Avanzados en Dirección de Empresas
}

Graduates' satisfaction with the training received in the Master of Advanced Studies in Business Administration

\author{
María del Mar González Zamora (mmgonza@us.es) \\ Carlos Sanchís Pedregosa (csanchis@us.es) \\ Universidad de Sevilla (España) \\ http://dx.doi.org/10.12795/EDUCADE.2014.105.04
}

\begin{abstract}
RESUMEN: Al objeto de conocer el nivel de satisfacción de los egresados con la formación recibida en el Máster de Estudios Avanzados en Dirección de Empresas, que se imparte en la Facultad de Ciencias Económicas y Empresariales de la Universidad de Sevilla, se ha realizado un estudio descriptivo de carácter transversal. Para ello se ha hecho uso de un cuestionario, al que han contestado los egresados que han culminado sus estudios hace al menos un año. Del análisis de los resultados se ha constatado, entre otras cuestiones, que, en opinión del alumnado: 1) el Máster contribuye en grados diferentes a la mejora de la formación investigadora y de la profesional, así como al acceso al mundo laboral y a las posibilidades de promoción profesional; 2) parece necesario mejorar en la capacitación de las competencias para llevarlas al nivel de lo que los alumnos han expresado como necesario en el plano práctico, identificándose aquellas competencias que deben priorizarse; 3) la mayoría de los alumnos están satisfechos de haber realizado el Máster, aunque la valoración dada al mismo parece demandar mejoras. Se han recibido propuestas realizadas al respecto por los egresados, que se han pasado a la coordinación del Máster con el objetivo de contribuir a la mejora continua del Título.
\end{abstract}

PALABRAS ClAVE: Postgrado, Estudios de Máster, Satisfacción con la formación recibida, Valoración competencias, Finalidad estudios, Mejora continua.

\begin{abstract}
In order to know the level of graduates' satisfaction related to the training received in the Master of Advanced Studies in Business Administration, taught at the Faculty of Economics and Business of the University of Seville, it was made a cross-descriptive study using a questionnaire, replied by graduates who finished their studies, at least, one year ago. The result analysis shows, among other things, that in the graduates' opinion: 1) the Master studies contributes in different levels to improve professional and research training, as well as gaining access to employment and having career opportunities, 2) it seems necessary to improve the competence training to bring them to the necessary level in professional skills, identifying those that should be improved as a priority, 3) most of the students are satisfied with the completion of this Master, but the results obtained seems to demand improvements, which are shown in this paper. Graduates have made proposals in this regard, that were passed to the coordination of the Master with the aim of contributing to the continuous improvement of Title.
\end{abstract}

KEYWORDS: Post graduate studies, Master studies, Satisfaction with the training received, Skills assessment, Purpose of the studies, Continuous improvement.

Artículo de Investigación. Recibido: 30-05-13 - Versión revisada: 04-09-13, Aceptado: 15-09-13, Publicado on-line 03-01-14 Licencia Creative Commons BY NC ND · 2014 · Asociación Española de Contabilidad y Administración de Empresas - AECA 


\section{INTRODUCCIÓN}

Según el artículo 10 del Real Decreto 1393/2007, de 29 de octubre, por el que se establece la ordenación de las enseñanzas universitarias oficiales, las enseñanzas de Máster tienen como finalidad la adquisición, por parte del estudiante, de una formación avanzada, de carácter especializado o multidisciplinar, orientada a la especialización académica o profesional, o bien a promover la iniciación en tareas investigadoras.

En este contexto, y desde el curso académico 2008/2009, se viene impartiendo el Máster de Estudios Avanzados en Dirección de Empresas. Se trata de un título oficial que se imparte en la Facultad de Ciencias Económicas y Empresariales de la Universidad de Sevilla.

El objetivo fundamental del citado Máster es atender, por una parte, las necesidades de formación de alto nivel de personas que pueden incorporarse o que ya están incorporadas a puestos de responsabilidad empresarial y, por otra, la formación de investigadores y doctores en los campos objeto de estudio.

Siguiendo a Segredo Pérez et al. (2004, p. 1), una de las formas de conocer si se cumple el objetivo anteriores a través del estudio del nivel de satisfacción de los egresados con la formación recibida. Son ellos los que, de acuerdo con su experiencia, podrán aportar información valiosa al respecto, llegando a contribuir a la mejora continua de dicho Máster. Hasta el momento, que conozcamos, ningún órgano perteneciente a la Universidad de Sevilla ha llevado a cabo dicho estudio, siendo el propósito de este trabajo.

"La satisfacción del alumno en los estudios universitarios ha cobrado vital importancia para las instituciones de este sector, pues de ella depende su supervivencia. Solo con la satisfacción de los alumnos se podrá alcanzar el éxito escolar, la permanencia de los estudiantes en la institución, y sobre todo, la formación de una valoración positiva boca a boca" Alves y Raposo (2005; citado en Salinas et al. (2008, p. 42)). Siguiendo a estos autores, consideramos que la satisfacción con la formación recibida se muestra aún más importante en los estudios de Máster. No se trata de que de ella dependa la supervivencia de la institución en la que se imparten dichos estudios (en nuestro caso, la Universidad de Sevilla), pero sí puede llegar a depender la supervivencia del propio título de Máster, dado el entorno actual en el que existe una amplísima oferta. Como ejemplo de ello, valga indicar que solo en la provincia de Sevilla se ofertan, en el área de la Dirección de Empresas, alrededor de una treintena de estudios de postgrado impartidos tanto por instituciones públicas como privadas (Emagister, 2013). Además, es conocida la creciente oferta de estudios de postgrado en modalidad a distancia y/u on-line, lo cual da una idea de la alta competencia que existe en el mercado de este tipo de estudios.

La satisfacción con la formación recibida es un concepto subjetivo, ya que depende del cumplimiento (o no) de las expectativas y deseos del alumnado (Athiyaman, 1997). Además, es un concepto amplio, cuyo estudio se ve condicionado por una gran cantidad de características, así como por la existencia de distintos puntos de vista para abordar su medición (Pérez Gil et al., 2010: 47-49).

Entre estos últimos, se encuentra la visión aportada por Lapeña y González (1996), que señalan que el grado de satisfacción de un alumno con la formación recibida está relacionado con su opinión sobre la misma (lo que pone de manifiesto la subjetividad antes comentada). Según estos autores, se trataría de ver en qué medida el alumno considera útil la formación en el desempeño de su trabajo o capacitación y si aquella le ha gustado. Esta es la perspectiva que seguiremos, y de ella derivan los objetivos fijados en el presente trabajo. 
De esta forma, nos planteamos analizar:

- La utilidad del Máster para alcanzar los fines que el alumnado persigue con su realización (no olvidemos el importante papel que juegan sus expectativas y deseos). En este sentido, nos hemos preguntado: zqué finalidad perseguía el alumnado al cursar el Máster? ¿̇Ha sido este de utilidad para alcanzar tales fines?

- La utilidad de la formación recibida en el Máster para el desempeño profesional/investigador del alumnado. Para medir esto nos hemos preguntado: ¿ha contribuido el Máster a que los egresados adquieran las competencias necesarias para su desempeño profesional/investigador?

- La opinión general que tienen los egresados sobre la formación recibida, de forma que intentamos responder a las siguientes cuestiones: ¿̇qué valoración general realizan los egresados de sus estudios de Máster? ¿̇stán satisfechos de haber realizado el Máster de Estudios Avanzados en Dirección de Empresas?

Para cubrir los objetivos anteriores, hemos recabado la opinión del alumnado mediante una encuesta que fue administrada on-line. Los detalles de la metodología se recogen más adelante, mostrándose posteriormente los resultados. El último apartado está destinado a una serie de consideraciones finales. Previo a todo ello, a continuación, detallamos el contexto en el que se ha llevado a cabo el presente trabajo.

\section{CONTEXTO}

Como se ha comentado en el apartado anterior, desde el curso 2008/09 se viene impartiendo el Máster de Estudios Avanzados en Dirección de Empresas, que en 2010/11 y tras superar el proceso de verificación, pasó a su denominación actual como Máster Universitario de Estudios Avanzados en Dirección de Empresas (ver figura 1).

Figura 1.Antecedentes del Máster de Estudios Avanzados en Dirección de Empresas

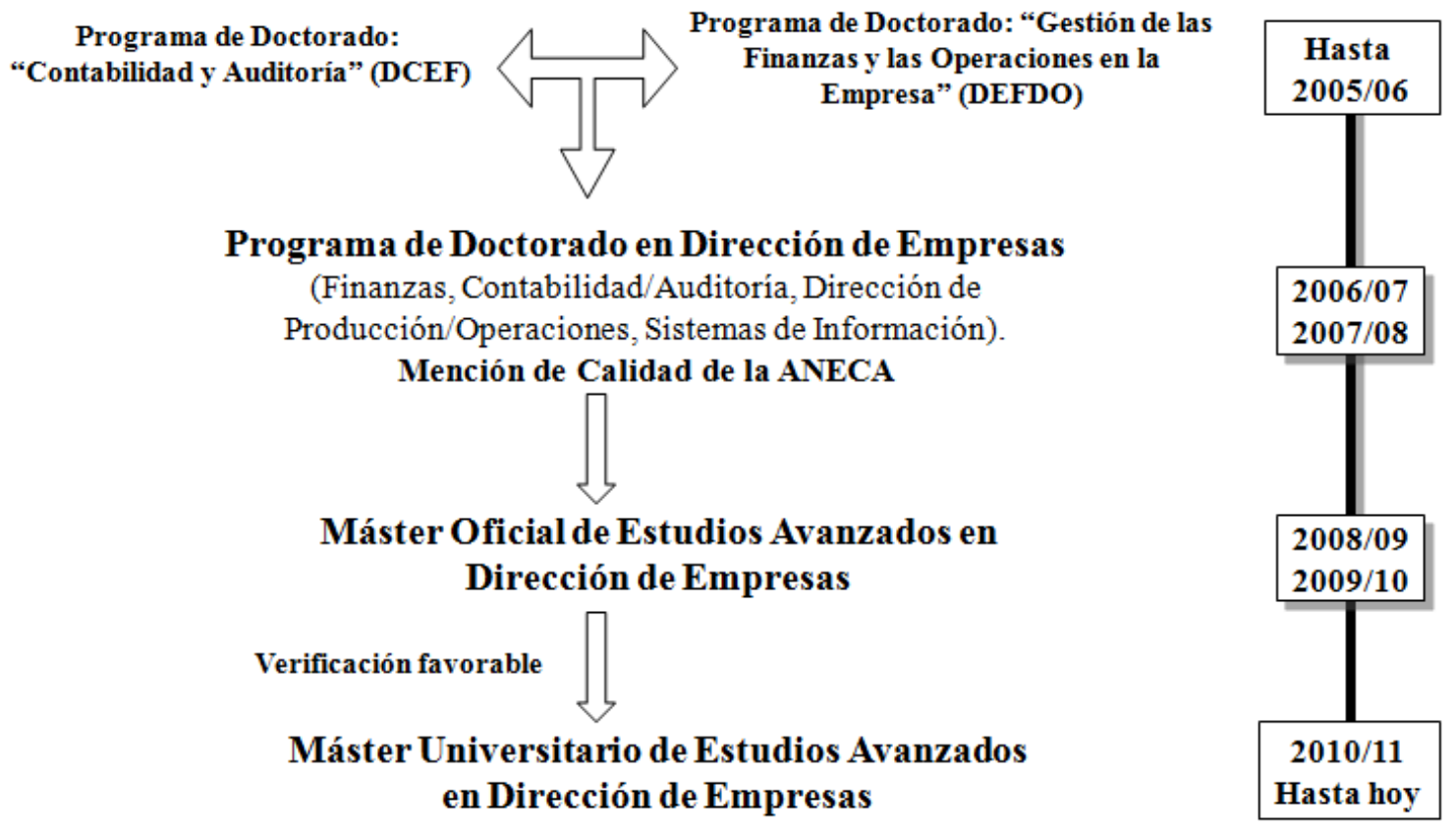

Fuente: Elaboración propia 
Como puede verse en la Figura 1, los antecedentes del mismo se remontan al curso 2006/2007, en el que comienza a impartirse el Programa de Doctorado en Dirección de Empresas. Este supuso la integración de los dos programas de doctorado que, hasta el momento, impartían los departamentos implicados en la docencia actual del Máster (el Dpto. de Contabilidad y Economía Financiera (DCEF) y el Dpto. de Economía Financiera y Dirección de Operaciones (DEFDO)).

Hay que tener presente que el Máster en el que centramos nuestra investigación es presencial.

Como se ha comentado en la introducción, su objetivo fundamental es atender, por una parte, las necesidades de formación de alto nivel de personas que pueden incorporarse o que ya están incorporadas a puestos de responsabilidad empresarial y, por otra, la formación de investigadores y doctores en los campos objeto de estudio (Dirección de Operaciones, Finanzas-Contabilidad/Auditoría y Sistemas/Tecnologías de Información). En este proceso, se pretende que el alumnado desarrolle una visión sistémica/interfuncional de la empresa, pero sin olvidar la especialización. Para ello, dicho Máster se ha organizado a través de una estructura de cursos comunes obligatorios, complementada con tres itinerarios de especialización: 1) Dirección de Producción/Operaciones-Sistemas/Tecnologías de Información (en adelante, DPO-STI); 2) Finanzas-Contabilidad/Auditoría (en adelante, $\mathrm{Fl}-\mathrm{CO}$ ); 3) Investigación (en adelante, INV).

Cualquiera que sea el itinerario elegido por el alumnado, éste debe cursar 60 créditos, 45 correspondientes a diversas asignaturas que, en su mayoría, dependen del itinerario seleccionado y los 15 restantes correspondientes al desarrollo de un Trabajo Fin de Máster.

En total, en el Máster se imparten 23 asignaturas (como se ha dicho anteriormente, algunas de ellas comunes a algunos de los itinerarios), cuyos créditos oscilan entre un máximo de 10 y un mínimo de 3. Para la impartición de las diferentes asignaturas se planifican clases, de entre 4 y 5 horas, en horario de tarde, impartiéndose, cada tarde, solo una asignatura.

Este Máster ha venido ofertando 25 plazas cada curso académico, aunque a partir del curso 2012-2013 las plazas han aumentado a 30.

\section{METODOLOGÍA}

Para la consecución de los objetivos del presente trabajo se ha realizado un estudio descriptivo de carácter transversal. Como técnica de recogida de datos se ha desarrollado un cuestionario, cuyo borrador ha sido posteriormente testado para su depuración.

El cuestionario completo constaba de un total de 30 preguntas divididas en 5 bloques. El primero de ellos estaba dedicado a recabar datos generales (como el itinerario cursado y los motivos por los que decidió cursar el Máster). El segundo bloque de preguntas giraba en torno a la situación laboral del alumnado tanto al comienzo del Máster como en el momento de completar la encuesta. El tercer bloque recoge la opinión del alumnado respecto a la utilidad del Máster en diferentes aspectos como acceso al mundo laboral o la mejora profesional. El cuarto bloque permite que el alumnado valore las competencias que necesitan en su trabajo y el grado de contribución del Máster a su desarrollo y, por último, el quinto bloque posibilita al alumnado realizar una valoración general de dicho Máster.

La administración del cuestionario se realizó vía Web, accediendo los egresados a él a través de un enlace que recibieron en sus direcciones de correo electrónico junto con 
un texto explicativo en que se les instaba a participar en el estudio, cumplimentando la encuesta.

Con vistas a tener una mayor perspectiva sobre la posible utilidad del Máster, la población objeto de estudio se ha centrado en el alumnado que, en el momento de realizar la encuesta, había culminado sus estudios de Máster hacía, al menos, un año. Como puede verse en la tabla 1, esto ha delimitado nuestra población objeto de estudio a 28 alumnos. De ellos han contestado a la encuesta 24, lo que supone una tasa de respuesta del $85,7 \%$ (similares valores en términos absoluto y relativo se han obtenido en otros trabajos relacionados con estudios de Máster, como por ejemplo, Segredo Pérez et al., (2004)). En la tabla 1 puede verse el desglose de alumnos por itinerario.

TABLA 1.POBLACIÓN Y ALUMNOS ENCUESTADOS POR ITINERARIO

\begin{tabular}{|c|c|c|c|c|}
\hline Itinerarios & DPO-STI & FI-CO & INV & TOTAL \\
\hline Población & $10(35,7 \%)$ & $6(21,4 \%)$ & $12(42,9 \%)$ & 28 \\
\hline Encuestados & $10(41,7 \%)$ & $3(12,5 \%)$ & $11(45,8 \%)$ & 24 \\
\hline
\end{tabular}

Fuente: Elaboración propia

\section{RESULTADOS}

A continuación mostramos los resultados obtenidos, exponiéndolos en función de los objetivos que se persiguen en el presente trabajo.

\subsection{Objetivo 1) analizar la utilidad del máster para alcanzar los fines que el alumnado PERSIGUE CON SU REALIZACIÓN}

Para contextualizar los resultados correspondientes a este primer objetivo, consideramos que es de interés tener presente la situación laboral del alumnado al inicio del Máster. Dicha situación la muestra la Tabla 2.

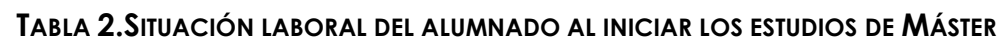

\begin{tabular}{|l|l|l|}
\hline Situación laboral & Alumnos & $\mathbf{\%}$ \\
\hline Desempleado & 7 & 29,2 \\
\hline Trabajador por cuenta propia & 0 & 0 \\
\hline Trabajador por cuenta ajena & 17 & 70,8 \\
\hline Total & $\mathbf{2 4}$ & \multirow{2}{|l|}{} \\
\cline { 1 - 2 } & &
\end{tabular}

Fuente: Elaboración propia

Tal como puede verse en la tabla 2 , el $29,2 \%$ del alumnado (7 de 24) estaba desempleado al iniciar sus estudios de Máster. El resto estaba empleado en la Administración Pública o en diferentes tipos de empresas.

Volviendo al primer objetivo, su análisis requiere conocer, por una parte, los motivos que llevaron al alumnado a cursar el Máster. Por otra parte, debemos conocer si, en 
opinión del alumnado, el Máster ha contribuido a la consecución de dichas finalidades. A continuación pasamos a ver ambas cuestiones.

\subsection{1 .Finalidades de cursar el Máster}

Preguntados los alumnos por la/s finalidad/es por la/s que realizaron los mencionados estudios, los resultados se muestran en la tabla 3. Hay que tener presente que, en la pregunta planteada, se permitió la selección de más de una respuesta, ello hace que el total de respuestas (35) sea mayor al número de alumnos encuestados (24).

TABLA 3.Finalidad CON LA QUe los alumnos Realizaron SUS ESTUdios de MÁster (I)

\begin{tabular}{|l|c|c|}
\hline Finalidad & $\mathbf{N}^{\circ}$ alumnos & $\mathbf{\%}$ \\
\hline Ampliar estudios & 19 & 54,3 \\
\hline Impulsar carrera profesional & 11 & 31,4 \\
\hline Otros: Realizar Tesis Doctoral & 3 & 8,6 \\
\hline Acceder al mundo laboral & 2 & 5,7 \\
\hline Total & 35 & \\
\cline { 1 - 3 } & &
\end{tabular}

Fuente: Elaboración propia

Como puede verse en la tabla 3, la mayoría de los alumnos indican que la finalidad con la que han cursado el Máster es la de ampliar estudios (54,3\%). En segundo lugar, aparece impulsar la carrera profesional $(31,4 \%)$, lo que no es de extrañar dado que, recordemos, la mayoría del alumnado (17, lo que supone un $70,8 \%$ ) estaba empleado en el momento de comenzar a cursar el Máster.

A gran distancia se encuentran la realización de una Tesis Doctoral $(8,6 \%)$ y el acceso al mundo laboral, que es señalada solo por el 5,7\% de los alumnos. Este último dato resulta sorprendente, ya que no todos los alumnos desempleados al comienzo del Máster marcan el acceso al mundo laboral como finalidad para la realización del mismo, solo lo hacen 2 de los 7 alumnos desempleados.

Como se ha comentado con anterioridad, en esta pregunta se permitió elegir más de una respuesta, mostrando la Tabla 4 las combinaciones de contestaciones dadas por los 24 alumnos encuestados. En ella puede verse cómo la mayor parte de los alumnos $(70,9 \%)$ realizan el Máster solo para ampliar estudios o bien para ampliar estudios esperando, además, impulsar su carrera profesional.

Según Sereno (2010) muchos estudiantes deciden realizar estudios de postgrado para tener más fácil el acceso al mundo laboral o para impulsar su carrera profesional. En nuestro caso, se comparte la afirmación anterior en lo que respecta al impulso de la carrera profesional. Sin embargo, en opinión de los alumnos encuestados, el tener más fácil el acceso al mundo laboral no es una de las principales motivaciones para cursar el Máster de Estudios Avanzados en Dirección de Empresas.

Entendemos que ello puede deberse a una cuestión de matiz, ya que consideramos que, aquellos alumnos que no estaban trabajando al inicio del Máster, lo realizaron con el fin inmediato de ampliar estudios con idea de, en un futuro más cercano que lejano, poder acceder al mercado laboral. Esto último parece corroborarse si miramos la situación laboral del alumnado en el momento de completar la encuesta. Solo 3 de 
los 7 alumnos que manifestaban estar desempleados al comienzo del Máster continúan en esa situación. No podemos asegurar que su acceso al mercado laboral se haya debido únicamente al hecho de cursar el Máster, aunque sería de interés conocer la opinión del alumnado a este respecto. A continuación se aborda esta cuestión, entre otras.

Tabla 4.Finalidad con la que los alumnos realizaron sus estudios de MÁster (II)

\begin{tabular}{|l|c|c|}
\hline Finalidad & $\begin{array}{c}\mathbf{N}^{\circ} \\
\text { alumnos }\end{array}$ & $\mathbf{\%}$ \\
\hline Ampliar estudios & 10 & 41,7 \\
\hline Ampliar estudios + Impulsar carrera profesional & 7 & 29,2 \\
\hline Impulsar carrera profesional & 2 & 8,3 \\
\hline Realizar Tesis Doctoral & 2 & 8,3 \\
\hline Acceder al mundo laboral & 1 & 4,2 \\
\hline $\begin{array}{l}\text { Ampliar estudios + Acceder al mundo laboral + Impulsar carrera } \\
\text { profesional }\end{array}$ & 1 & 4,2 \\
\hline Ampliar estudios + Impulsar carrera profesional + Realizar Tesis Doctoral & $\mathbf{2 4}$ & \\
\hline Total & 1 & 1 \\
\cline { 1 - 3 }
\end{tabular}

Fuente: Elaboración propia

\subsubsection{Utilidad del Máster a la consecución de las finalidades de los egresados}

Para valorar dicha utilidad hemos preguntado al alumnado su opinión sobre si la realización del Máster ha contribuido a mejorar: 1) su formación, 2) las posibilidades de acceso al mundo laboral y 3) las posibilidades de promoción laboral. Como puede verse, estos tres aspectos están en clara relación con las finalidades mencionadas anteriormente. A continuación, pasamos a comentar los resultados obtenidos.

\subsubsection{Contribución del Máster a la mejora de la formación del alumnado}

Dado que el Máster tiene dos tipos de alumnos diferenciados, aquellos que optan por un itinerario de tipo profesional (itinerarios DPO-STI y FI-CO) y aquellos que se orientan por el itinerario de Investigación, se decidió preguntar a ambos grupos por separado si el Máster ha contribuido a mejorar su formación (profesional o investigadora, según el caso). Los resultados, en valores absolutos y relativos, pueden verse en la tabla 5 , teniendo presente que se ha empleado una escala de 1 a 5 (1= totalmente en desacuerdo, 5 = totalmente de acuerdo).

Como puede verse en la tabla 5 , el $91 \%$ de los egresados que han cursado el itinerario de investigación consideran que el Máster ha contribuido a mejorar su formación investigadora. Tan solo 1 alumno (9\%) está en desacuerdo con dicha afirmación. Sin embargo, la situación cambia cuando se le pregunta a los alumnos de perfil profesional. En este caso, solo el $38,5 \%$ de los alumnos consideran que el Máster ha contribuido a mejorar su formación profesional. El mismo porcentaje de alumnos opinan que el máster no ha contribuido a la mencionada mejora. 
Tabla 5.CONtribución del Máster a la formación del egresado

\begin{tabular}{|c|c|c|c|c|c|c|c|}
\hline & 1 & 2 & 3 & 4 & 5 & Media & D.T. \\
\hline $\begin{array}{l}\text { El Máster ha } \\
\text { contribuido a } \\
\text { mejorar mi } \\
\text { formación } \\
\text { profesional }\end{array}$ & $\begin{array}{c}0 \\
(0 \%)\end{array}$ & $\begin{array}{c}5 \\
(38,5 \%)\end{array}$ & $\begin{array}{c}3 \\
(23,1 \%)\end{array}$ & $\begin{array}{c}3 \\
(23,1 \%)\end{array}$ & $\begin{array}{c}2 \\
(15,4 \%)\end{array}$ & 3,15 & 1,14 \\
\hline $\begin{array}{l}\text { El Máster ha } \\
\text { contribuido a } \\
\text { mejorar mi } \\
\text { formación } \\
\text { investigadora }\end{array}$ & $\begin{array}{c}0 \\
(0 \%)\end{array}$ & $\begin{array}{c}1 \\
(9 \%)\end{array}$ & $\begin{array}{c}0 \\
(0 \%)\end{array}$ & $\begin{array}{c}5 \\
(45,5 \%)\end{array}$ & $\begin{array}{c}5 \\
(45,5 \%)\end{array}$ & 4,27 & 0,90 \\
\hline
\end{tabular}

Fuente: Elaboración propia

Consideramos que estos resultados dejan entrever el pasado del Máster como Programa de Doctorado (tal como se comentó en el apartado 2), siendo necesaria la reorientación de algunos contenidos al objeto de que estos contribuyan, en mayor medida, a la mejora profesional de los alumnos que opten por los itinerarios de DPO-STI o de Fl-CO.

\subsubsection{Contribución del Máster a las posibilidades de acceso al mundo laboral}

En la Tabla 6 se muestran los resultados obtenidos al preguntar, a los alumnos que manifestaban estar desempleados al inicio del Máster, sobre si dichos estudios han influido o influyen en las posibilidades de acceso al mundo laboral.

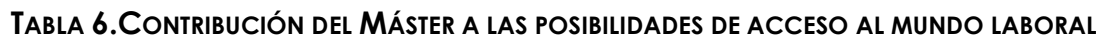

\begin{tabular}{|c|c|c|c|c|c|c|c|}
\cline { 2 - 7 } \multicolumn{1}{c|}{} & $\mathbf{1}$ & $\mathbf{2}$ & $\mathbf{3}$ & $\mathbf{4}$ & $\mathbf{5}$ & Media & D.T. \\
\hline $\begin{array}{c}\text { Mis estudios de } \\
\text { Máster han } \\
\text { influido/influyen en } \\
\text { mis posibilidades de } \\
\text { acceso al mundo } \\
\text { laboral }\end{array}$ & 1 & 0 & 1 & 4 & 1 & 3,57 & 1,27 \\
\hline
\end{tabular}

Fuente: Elaboración propia

Como puede verse en la tabla 6 , el $71,4 \%$ de los alumnos se muestra de acuerdo 0 totalmente de acuerdo con la afirmación anterior, ello a pesar de que, recordemos, el acceso al mundo laboral no era una de las principales finalidades por la que los alumnos desempleados cursaron el Máster.

\subsubsection{Contribución del Máster a las posibilidades de promoción laboral}

Por último, preguntamos a los alumnos que manifestaban estar empleados en el momento de realizar la encuesta, su opinión acerca de si el Máster ha influido/influye en sus posibilidades de promoción laboral dentro o fuera de la empresa actual. La tabla 7 recoge los resultados, en los que puede verse que solo el 14,2\% del alumnado se muestra en desacuerdo con dicha afirmación. 
La opinión del resto se reparte, por igual (un 42,9\%), entre los que consideran que sí ha contribuido y aquellos otros que no están ni de acuerdo ni en desacuerdo con dicha afirmación. En estos resultados habría que tener en cuenta que el $70 \%$ de los alumnos que contestaron a esta pregunta manifestaban tener expectativas laborales de promoción.

tabla 7.Contribución del Máster a las posibilidades de promoción laboral (I)

\begin{tabular}{|c|c|c|c|c|c|c|c|}
\cline { 2 - 7 } \multicolumn{1}{c|}{} & $\mathbf{1}$ & $\mathbf{2}$ & $\mathbf{3}$ & $\mathbf{4}$ & $\mathbf{5}$ & Media & D.T. \\
\hline $\begin{array}{c}\text { Mis estudios de } \\
\text { Máster han } \\
\text { influido/influyen } \\
\text { en mis } \\
\text { posibilidades } \\
\text { de promoción } \\
\text { laboral dentro } \\
\text { de mi empresa } \\
\text { ofuera de la } \\
\text { misma }\end{array}$ & 2 & 1 & 9 & 3 & 6 & 3,44 & 1,29 \\
\hline
\end{tabular}

Fuente: Elaboración propia

Si nos centramos en los 11 alumnos que, según la Tabla 3, señalaban como finalidad para la realización del Máster el impulso a su carrera profesional, la tabla 8 muestra los resultados obtenidos. Como puede verse, para el conjunto de estos alumnos el valor medio aumenta hasta el 3,60, disminuyendo el porcentaje tanto de aquellos que muestran su desacuerdo con la afirmación como de los que se muestran de acuerdo o muy de acuerdo con ella, y aumentando el porcentaje de los alumnos que no están ni de acuerdo ni en desacuerdo con dicha afirmación.

Tabla 8.Contribución del Máster a las posibilidades de promoción laboral (II)

\begin{tabular}{|c|c|c|c|c|c|c|c|c|}
\cline { 2 - 8 } \multicolumn{1}{c|}{} & $\mathbf{1}$ & $\mathbf{2}$ & $\mathbf{3}$ & $\mathbf{4}$ & $\mathbf{5}$ & NS/NC & Media & D.T. \\
\hline $\begin{array}{c}\text { Mis estudios } \\
\text { de Máster } \\
\text { han influido } \\
\text { o influyen en } \\
\text { mis } \\
\text { posibilidades } \\
\text { de } \\
\text { promoción } \\
\text { laboral } \\
\text { dentro de mi } \\
\text { empresa o } \\
\text { fuera de la } \\
\text { misma }\end{array}$ & 0 & 1 & 5 & 1 & 3 & 1 & 3,60 & 1,07 \\
\hline
\end{tabular}

Fuente: Elaboración propia 
Una vez terminado de exponer los resultados correspondientes al primer objetivo, pasamos al análisis del segundo.

\subsection{Objetivo 2) anAlizar la utilidad de la fOrmación ReCibida en el máster para el deSEMPeÑo PROFESIONAL/ INVESTIGADOR DEL EGRESADO}

Para analizar dicha utilidad nos hemos centrado en el estudio de las competencias, ya que estas muestran la preparación de los egresados para su participación en el mercado laboral (bien sea como profesionales o como investigadores) (Allen et al. (2003: 31) y Rubin y Dierdorff (2009: 210)).

Las competencias generales perseguidas en el Máster se muestran en su memoria de verificación. Para la realización del presente trabajo, se han completado dichas competencias con las recogidas en el Real Decreto 1393/2007 (que establece la ordenación de las enseñanzas universitarias oficiales) y también con las que figuran en el non-nato Marco Español de Cualificaciones para la Educación Superior (MECES). El conjunto de las competencias objeto de análisis se muestra en la primera columna de la Tabla 9.

Acerca de cada una de estas competencias se preguntó al alumnado, que actualmente está trabajando, su opinión sobre la necesidad que de ellas tienen a la hora de realizar su trabajo, así como la contribución del Máster a su desarrollo, empleando para ello una escala de 1 a 5 (1= necesidad/contribución nula, $5=$ necesidad/contribución muy alta). Los valores medios y las desviaciones típicas obtenidas pueden verse en la Tabla 9.

A juzgar por el valor medio del nivel necesario en el trabajo de cada una de las de las competencias que se persiguen en el Máster, aunque sería necesario precisar aquellas que parecen necesitar una mayor atención.

Para tal fin, y para cada competencia, se ha calculado la diferencia entre el valor medio de su nivel necesario en el trabajo y el de la contribución del Máster a su desarrollo, observándose, también con ayuda de la tabla 9, cómo hay seis de ellas que presentan diferencias estadísticamente significativas (señaladas en negrita). Estas serían, en opinión del alumnado, las principales competencias que deberían reforzarse en el Máster.

\subsection{Objetivo 3) ReCabar la opinión General que tienen lOS Egresados sobre la fORMación RECIBIDA}

Aunque de los resultados comentados previamente se pueden extraer conclusiones sobre el grado de satisfacción de los alumnos tras la realización del Máster, hemos querido preguntar directamente a ellos sobre esta cuestión, empleando una escala 1 a 5 ( 1 = nada satisfecho; $5=$ muy satisfecho).

Como puede verse en la Tabla 10, la mayoría del alumnado $(58,34 \%)$ contesta indicando que está satisfecho o muy satisfecho. Aunque no se puede obviar que la moda se encuentra en el valor medio de la escala, siendo elevado el porcentaje de alumnos $(37,5 \%)$ que no están ni satisfechos ni insatisfechos con la realización del Máster. 


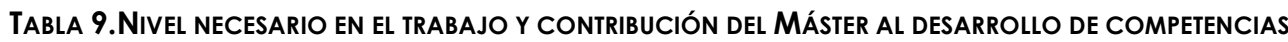

\begin{tabular}{|c|c|c|c|c|}
\hline & \multicolumn{2}{|c|}{$\begin{array}{l}\text { Nivel necesario } \\
\text { en el trabajo }\end{array}$} & \multicolumn{2}{|c|}{$\begin{array}{l}\text { Contribución del } \\
\text { Máster a su } \\
\text { desarrollo }\end{array}$} \\
\hline & Media & D.T. & Media & D.T. \\
\hline $\begin{array}{l}\text { Profundización en las materias objeto de estudio } \\
\text { mediante un enfoque integrado }\end{array}$ & 3,50 & 1,01 & 3,21 & 1,10 \\
\hline Capacidad para trabajo en equipo (*) & 4,32 & 1,09 & 3,63 & 1,01 \\
\hline $\begin{array}{l}\text { Capacidad para tratar situaciones complejas y } \\
\text { formular juicios argumentados }\end{array}$ & 4,09 & 0,87 & 3,67 & 0,96 \\
\hline Capacidad para tomar decisiones (**) & 4,23 & 0,87 & 3,46 & 0,83 \\
\hline Capacidad de análisis y síntesis & 4,27 & 0,77 & 3,88 & 1,08 \\
\hline $\begin{array}{l}\text { Capacidad para aplicar en la práctica los } \\
\text { conocimientos adquiridos }\end{array}$ & 3,91 & 0,75 & 3,38 & 1,01 \\
\hline Capacidad de adaptación a nuevas situaciones $\left({ }^{*}\right)$ & 4,32 & 0,78 & 3,58 & 0,93 \\
\hline Compromiso ético en el trabajo (**) & 3,77 & 1,15 & 2,92 & 1,02 \\
\hline $\begin{array}{l}\text { Sensibilidad hacia temas medioambientales y } \\
\text { sociales }\end{array}$ & 3,05 & 1,21 & 2,67 & 0,96 \\
\hline Capacidad de trabajo bajo presión (**) & 4,55 & 0,80 & 3,42 & 1,18 \\
\hline Comunicación oral y escrita & 4,27 & 0,88 & 3,75 & 0,99 \\
\hline $\begin{array}{l}\text { Habilidad para analizar y emplear información de } \\
\text { diversas fuentes }\end{array}$ & 4,18 & 0,85 & 4,00 & 0,93 \\
\hline Habilidad para la búsqueda de información & 4,32 & 0,99 & 4,04 & 1,08 \\
\hline Capacidad de aprendizaje autónomo & 4,23 & 0,87 & 3,58 & 0,93 \\
\hline Capacidad de integrar conocimientos & 4,18 & 0,80 & 3,71 & 0,86 \\
\hline $\begin{array}{l}\text { Capacidad de resolución de problemas en } \\
\text { entornos nuevos o poco conocidos (*) }\end{array}$ & 4,32 & 0,89 & 3,46 & 0,98 \\
\hline $\begin{array}{l}\text { Comunicar conclusiones (y los conocimientos y } \\
\text { razones últimas que las sustentan) de un modo } \\
\text { claro }\end{array}$ & 4,05 & 1,05 & 3,83 & 0,96 \\
\hline Capacidad para desarrollar y aplicar ideas & 4,14 & 0,94 & 3,67 & 0,87 \\
\hline $\begin{array}{l}\text { Capacidad de concebir, diseñar y poner en } \\
\text { práctica un proceso de investigación con seriedad } \\
\text { académica }\end{array}$ & 3,09 & 1,659 & 3,50 & 1,50 \\
\hline $\begin{array}{l}\text { Capacidad para estructurar y analizar problemas; } \\
\text { generar hipótesis de investigación y utilizar métodos } \\
\text { para su contraste }\end{array}$ & 3,18 & 1,563 & 3,21 & 1,02 \\
\hline
\end{tabular}

(*) Implica que se rechaza que la media del nivel necesario de cada competencia y la contribución del Máster a su desarrollo sea la misma con una significación del 5\%.

$\left.{ }^{* *}\right)$ Lo mismo con una significación del $1 \%$.

Fuente: Elaboración propia 
Ma del Mar González, Carlos Sanchís Satisfacción de los egresados con la formación recibida en el MEADE

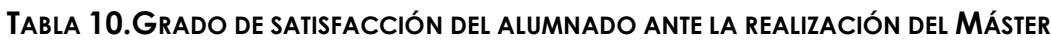

\begin{tabular}{|c|c|c|c|c|c|c|c|}
\cline { 2 - 7 } \multicolumn{1}{c|}{} & $\mathbf{1}$ & $\mathbf{2}$ & $\mathbf{3}$ & $\mathbf{4}$ & $\mathbf{5}$ & Media & D.T. \\
\hline $\begin{array}{c}\text { ¿̇Está satisfecho de } \\
\text { haber realizado el } \\
\begin{array}{c}\text { Máster de Estudios } \\
\text { Avanzados en } \\
\text { Dirección de } \\
\text { Empresas? }\end{array}\end{array}$ & 0 & 1 & 9 & 7 & 7 & 3,83 & 0,92 \\
\hline
\end{tabular}

Fuente: Elaboración propia

Por otra parte, también preguntamos al alumnado la valoración general que tienen acerca del Máster de Estudios Avanzados en Dirección de Empresas. Los resultados se muestran en la tabla 11, en la que se ha empleado, de nuevo, una escala de 1 a 5 (indicando 1 una valoración muy mala y 5 una valoración muy buena). Como puede verse, en este caso, hay el mismo porcentaje de alumnos $(45,8 \%)$ que valoran al Máster de forma buena o muy buena que aquellos que no lo consideran ni malo ni bueno.

Tabla 11.Valoración general del alumnado hacia el Máster de Estudios Avanzados en Dirección de Empresas

\begin{tabular}{|c|c|c|c|c|c|c|c|}
\hline & 1 & 2 & 3 & 4 & 5 & Media & D.T. \\
\hline $\begin{array}{c}\text { Desde su posición } \\
\text { de egresado, } \\
\text { ¿̇cuál es su } \\
\text { valoración } \\
\text { general del } \\
\text { Máster? }\end{array}$ & $\begin{array}{c}1 \\
(4,17 \%)\end{array}$ & $\begin{array}{c}1 \\
(4,17 \%)\end{array}$ & $\begin{array}{c}11 \\
(45,83 \%)\end{array}$ & $\begin{array}{c}7 \\
(29,17 \%)\end{array}$ & $\begin{array}{c}4 \\
(16,67 \%)\end{array}$ & 3,5 & 0,98 \\
\hline
\end{tabular}

Fuente: Elaboración propia

Aunque, en general, esta valoración no se puede considerar negativa, sí parece demandar mejoras. Sobre estas preguntamos a los egresados, haciendo uso de una pregunta abierta. Dichas mejoras se pueden agrupar en distintos ámbitos, como puede verse en la Tabla 12.

Si bien coincidimos con algunas de las acciones sugeridas por el alumnado (como puede ser el fomento del método del caso), sin embargo, no respaldamos otras, como la solicitud de un temario desarrollado de las asignaturas. Entendemos que esto último iría en contra de algunas de las competencias del Máster (ver tabla 9) como son: la Capacidad de análisis y síntesis, la Habilidad para analizar y emplear información de diversas fuentes, la Habilidad para la búsqueda de información y la Capacidad de integrar conocimientos.

Esto pone de manifiesto la necesidad de abordar un proceso de reflexión sobre la base de las distintas mejoras propuestas y los distintos ámbitos contenidos en la tabla 12. Entendemos que esta reflexión compete a los órganos de gestión académica del Máster, que son los que podrán poner en práctica aquellas propuestas que se consideran que redundarán en una mejora del mismo. Para hacer posible ello, estas mejoras propuestas por los alumnos han sido puestas en conocimiento de la Coordinación del Máster. 
TABLA 12.PRINCIPALES MEJORAS SUGERIDAS POR LOS ALUMNOS

\begin{tabular}{|c|c|}
\hline Ámbito de la mejora & Mejoras sugeridas por el alumnado \\
\hline Plan de estudios & - Incluir prácticas profesionales 1. \\
\hline Planificación docente & $\begin{array}{l}\text { - Reducir el número de horas de clase diaria de cada } \\
\text { asignatura². }\end{array}$ \\
\hline $\begin{array}{l}\text { Organización de las } \\
\text { asignaturas }\end{array}$ & $\begin{array}{l}\text { - Mejorar la coordinación de aquellas asignaturas que tienen } \\
\text { un elevado número de profesores. } \\
\text { - } \quad \text { Aumentar el número de seminarios o sesiones impartidas por } \\
\text { profesionales. } \\
\text { - Invitar, en cada asignatura, a un profesional que dé una } \\
\text { visión práctica de cómo se aplica en el mundo laboral los } \\
\text { conocimientos adquiridos. }\end{array}$ \\
\hline $\begin{array}{l}\text { Contenido de las } \\
\text { asignaturas }\end{array}$ & $\begin{array}{l}\text { - } \quad \text { Mayor enfoque al mundo empresarial. } \\
\text { - } \quad \text { Aumentar contenido práctico. }\end{array}$ \\
\hline $\begin{array}{l}\text { Metodología de las } \\
\text { asignaturas }\end{array}$ & $\begin{array}{l}\text { - } \quad \text { Fomentar el trabajo en equipo en clase. } \\
\text { - } \quad \text { Realizar más trabajos donde se apliquen los conocimientos } \\
\text { adquiridos en casos de estudio. }\end{array}$ \\
\hline $\begin{array}{l}\text { Material de las clases y } \\
\text { su difusión }\end{array}$ & $\begin{array}{l}\text { - Proporcionar temario desarrollado de las asignaturas. } \\
\text { - Disponer de un sitio donde se integren el material y } \\
\text { contenido de todas las asignaturas }{ }^{4} \text {. }\end{array}$ \\
\hline Evaluación asignaturas & $\begin{array}{l}\text { - } \text { Mayor flexibilidad en la asistencia a clases } 5 \\
\text { - } \text { hamentar el nivel de exigencia, haciendo exámenes que } \\
\text { - } \quad \text { Reconsiderar las actividades de evaluación en algunas } \\
\text { asignaturas (se exigen demasiados trabajos en relación a } \\
\text { las clases/duración de las mismas). }\end{array}$ \\
\hline
\end{tabular}

Fuente: Elaboración propia

\section{CONSIDERACIONES FINALES}

1 Aunque recordemos que la mayoría de los estudiantes encuestados trabajaban en el momento de comenzar el Máster.

2 Recordemos que, tal como se comentó en el apartado relacionado con el contexto del trabajo, en general, cada tarde se planifican clases de entre 4 y 5 horas correspondientes a una única asignatura.

3 Recordemos que esta capacidad era una de las que necesitaba ser mejorada, ver Tabla 9.

4 Esto se ha conseguido en las últimas ediciones del Máster, al contar todas las asignaturas con presencia en la plataforma de enseñanza virtual empleada por la Universidad de Sevilla.

5 Dado que, como se dijo en el apartado 2, el Máster es presencial, la asistencia a clase es considerada a efectos de evaluación del alumnado. 
Tras la realización del estudio llevado a cabo con los egresados del Máster de Estudios Avanzados en Dirección de Empresas que, en el momento de realizar la encuesta, habían concluido sus estudios al menos hacía un año, hemos podido constatar lo siguiente:

- Los alumnos cursan el Máster fundamentalmente para ampliar estudios y para impulsar su carrera profesional.

- La gran mayoría de los alumnos que han cursado el itinerario de Investigación considera que el Máster ha contribuido a mejorar su formación investigadora.

- Sin embargo, la opinión del alumnado que ha cursado los itinerarios profesionales (DPO-STI; FI-CO) no es tan clara respecto a la contribución del Máster a la mejora de la formación profesional.

- La mayoría de los alumnos desempleados al comienzo del Máster considera que este ha contribuido/contribuye en sus posibilidades de acceso al mundo laboral.

- No hay mayoría de alumnos, actualmente trabajando, que opinen que sus estudios de Máster hayan contribuido o contribuyan en sus posibilidades de promoción (a pesar de que el $70 \%$ de ellos consideran que tienen expectativas laborales de promoción).

- Parece necesario mejorar en el desarrollo de las competencias para llevarlas al nivel de lo que los alumnos han expresado como necesario en el plano práctico. De forma prioritaria, y en opinión de los egresados, se debería mejorar la capacitación en las siguientes competencias:

- Capacidad para trabajo en equipo.

- Capacidad para tomar decisiones.

- Capacidad de adaptación a nuevas situaciones.

- Compromiso ético en el trabajo.

- Capacidad de trabajo bajo presión.

- Capacidad de resolución de problemas en entornos nuevos o poco conocidos.

- La mayoría de los alumnos están satisfechos de haber realizado el Máster, pero la valoración dada al mismo parece demandar mejoras. Se han recibido propuestas realizadas al respecto por los egresados, que se han pasado a la coordinación del Máster con el objetivo de contribuir a la mejora del mismo.

A tenor de los resultados obtenidos, parece clara la necesidad de potenciar el enfoque profesional de los estudios del Máster objeto del presente trabajo (algunas de las mejoras propuestas por los alumnos van encaminadas a ello- ver tabla 12). A este respecto, hay que decir que parte del profesorado del Máster tiene vinculación con el ámbito empresarial, aunque no puede negarse que dicha parte es pequeña. Es por ello que, desde la primera edición del Máster, se ha contado con la presencia de algunos profesionales que imparten seminarios sobre diversas temáticas, en un esfuerzo de mayor conexión con el mundo profesional. Sin embargo, en opinión del alumnado, parece que dicho esfuerzo no ha sido suficiente. Entre otros factores, el entorno actual, en el que cada vez es más difícil conseguir recursos, obstaculiza la generalización de la práctica antes mencionada. Siendo así, la situación actual obliga a un empleo más eficiente de los recursos y capacidades con los que cuenta la Universidad. A este respecto, no debemos obviar a los Profesores Asociados, cuya experiencia profesional puede convertirse en un gran valor añadido para los estudios de Máster. Sin embargo, 
las directrices actuales de la Universidad de Sevilla en materia de elaboración del plan de asignación del profesorado a los planes de organización docente dificulta el acceso a la docencia en Máster por parte de dicha categoría de profesoradob. Consideramos que una flexibilización de dichas directrices puede contribuir a un mejor aprovechamiento de las capacidades del personal de la Universidad, sin que haya necesidad (o, al menos, disminuyéndola) de buscar "fuera" la experiencia que muy probablemente ya tenemos "dentro".

Consideramos, además, que esta mayor conexión con el mundo profesional también podría lograrse en el desarrollo de Trabajos Fin de Máster. Actualmente, la normativa de Trabajos Fin de Carrera de la Universidad de Sevilla establece que el tutor será un profesor con plena capacidad docente? ${ }^{7}$. Atendiendo a la literalidad de dicha normativa, no pueden asumir funciones de tutela (o co-tutela) de Trabajos Fin de Máster ni los Profesores Asociados, a los que antes hemos hecho mención, ni tampoco profesionales sin vinculación con la Universidad. De nuevo, consideramos que una flexibilización de la normativa, estableciéndose de forma explícita la posibilidad de que profesionales y Profesores Asociados co-tutelen Trabajos Fin de Máster, puede añadir valor a los estudios de Máster con orientación profesional, mejorando, además, el carácter práctico de los mismos.

Una de las principales limitaciones del presente estudio la constituye el escaso número de alumnos encuestados (y de aquellos que cumplen los requisitos para ser encuestados). En este sentido, es nuestro propósito seguir encuestando a los alumnos de cursos posteriores que cumplan con el requisito de haber terminado el Máster, al menos, con un año de antelación a la cumplimentación de la encuesta. Ello también permitirá ver si con el paso del tiempo, efectivamente, se produce una mejora en la satisfacción del alumnado con respecto al título.

Por otra parte, consideramos que estos resultados obtenidos ganarían una mayor dimensión al compararlo con los de otros Máster afines impartidos tanto en la Universidad de Sevilla como fuera de ella. A este respecto, no hemos encontrado trabajos similares en nuestro campo de estudio que permitan realizar dicha comparativa. Sí hemos encontrado un estudio similar (aunque solo centrado en el análisis de las competencias), pero referido al ámbito de la sanidad (Segredo Pérez et al., 2004), por lo que los resultados no son comparables con los nuestros. En la medida en que se publiquen estudios similares, la comparativa de resultados también formarán parte de la continuidad del presente trabajo.

\section{BIBLIOGRAFÍA}

Allen, J.; RAMAeKeRs, G. y VAN Der VelDen, R. [2003]: La medición de las competencias de los titulados superiores. En J. Vidal García (ed.). Métodos de análisis de la inserción laboral de los universitarios: 31-54.

Alves H. y RAPOSO M. [2005]: La Medición de la Satisfacción en la Enseñanza Universitaria: El ejemplo de la Universidade da Beira Interior. Universidade da

6 La directriz $n^{\circ} 8$ del Reglamento para la elaboración de los Planes de Asignación de Profesorado a los Planes de Organización Docente (Acuerdo 3.2/C.G. 9-5-05) señala que: En la medida en que las posibilidades lo permitan, las enseñanzas de segundo ciclo serán asignadas a profesores doctores.

7 Artículo 5.1 de la Normativa Reguladora de los Trabajos Fin de Carrera de la Universidad de Sevilla. BOUS núm. 1, de 18 de enero de 2010. 
Beira Interior. Dpto. de Gestão e Economia, Covilhã (Portugal), http://128.118.178.162/eps/hew/papers/0511/0511004.pdf.

ATHIYAMAN, A. [1997]: Linking student satisfaction and service quality perceptions: the case of university education, European Journal of Marketing, vol.31, núm.7: 528-540.

EMAGISTER [2013]: http://Www.emagister.com

Lapeña lapeña, A. y González SÁnChez, M.C. [1996]: La formación continua de los trabajadores: manual del formador / [autores, Equipo IFES,]. Madrid Instituto de Formación y Estudios Sociales.

Pérez Gil, J.A.; LOzAno lozano, J.A.; Gómez De Terreros Guardiola, M. y Aguilera Jiménez, A. [2010]: Diseño de un instrumento para la evaluación de la satisfacción de la formación recibida de las diferentes asignaturas correspondientes al plan de estudios del grado en psicología de la Universidad de Sevilla, Revista de Enseñanza Universitaria, vol.36: 45-61.

REAL DECRETO 1393/2007, de 29 de octubre, por el que se establece la ordenación de las enseñanzas universitarias oficiales. Ministerio de Educación y Ciencia. BOE núm. 260, de 30 de octubre.

RUBIN, R.S. Y DIERDORFFF, E.C. [2009]: How relevant is the MBA? Assessing the alignment of required curricula and required managerial competencies, Academy of Management Learning \& Education, vol.8, núm.2: 208-224.

Salinas GutiérRez, A.; MORAles LOZANO, J.A. y MARTínez CAMBlOR, P. [2008]: Satisfacción del estudiante y calidad universitaria: un análisis explicatorio en la unidad académica multidisciplinaria agronomía y ciencias de la Universidad Autónoma de Tamaulipas, México, Revista de Enseñanza Universitaria, vol.31: 39-55.

Segredo Pérez, A.M.; Perdomo Victoria, I.; Pérez Piñero, J. y lópez Puig, P. [2004]: Satisfacción de los egresados de la Maestría de APS con las competencias adquiridas, Revista (online) Cubana de Medicina General Integral, vol.20, núm.2.

SERENO, E. [2010]: Tener estudios de postgrado abre las puertas a un 5\% más de ofertas de empleo, http://apms.es/183Yhhu 